\title{
'Hongbaleng', a Crabapple Cultivar with Large Fruit and Full-coverage Red Color
}

\author{
Jiayi Ji, Zhenglin Li, Ji Tian, Jie Zhang, Yanfen Lu, and \\ Xiaoxiao Qin
}

Beijing Advanced Innovation Center for Tree Breeding by Molecular Design, Beijing University of Agriculture, Beijing, 102206, China; Plant Science and Technology College, Beijing University of Agriculture, Beijing 102206, China; and Beijing Key Laboratory for Agricultural Application and New Technique, Beijing University of Agriculture, Beijing, 102206, China

\section{Jianjun Li \\ Yanqing District Fruit Technology Management Station, Beijing 10102104, China}

\section{Liqiang Liu}

Xishan Coal Electricity Group Co., Ltd. Environmental Protection \& Landscaping Bureau, Shanxi 030053, China

\section{Zhe Gao}

College of Food Science and Technology, Agriculture University of Hebei, Baoding 071000, China

\section{Yujing Hu, and Yuncong Yao}

Beijing Advanced Innovation Center for Tree Breeding by Molecular Design, Beijing University of Agriculture, Beijing, 102206, China; Plant Science and Technology College, Beijing University of Agriculture, Beijing 102206, China; and Beijing Key Laboratory for Agricultural Application and New Technique, Beijing University of Agriculture, Beijing 102206, China

Ornamental crabapple is a popular landscaping plant worldwide; it belongs to the Malus genus of the Rosaceae family. Ornamental crabapple has been used extensively in gardens, street plantings, and is an important ornamental tree due to its colorful leaves, flowers, fruits, and beautiful tree habit; it has also been used in apple cultivation as a rootstock and pollinator due to its wide adaptability and

Received for publication 12 May 2021. Accepted for publication 9 June 2021.

Published online 23 July 2021.

J.J. and Z.L. contributed equally to this work.

This work was financially supported by the National Natural Science Foundation of China (31901997), the General Project of the Scientific Research Program of the Beijing Municipal Commission of Education (KM202010020013), the Special Project of Rural Industry Revitalization supported by Beijing Municipal Commission of Science and Technology (Z201100008020007), the Beijing Advanced Innovation Center for Tree Breeding by Molecular Design, Beijing University of Agriculture.

Y.H. and Y.Y. are the corresponding authors. E-mail: 805756676@qq.com or yaoyc_20@126.com.

This is an open access article distributed under the CC BY-NC-ND license (https://creativecommons. org/licenses/by-nc-nd/4.0/). abundant pollen production (Dudash et al., 2011; Henry, 2007). In addition, its fruits and leaves are processed and used as dried fruit, in drinks, and also medicine because of their special nutritional and medicinal ingredients (Qian and Tang, 2005; Wang, 1989). Ongoing ornamental crabapple breeding has focused on developing a diversity of new varieties (Qian, 2005), which proposes an emphasis on varieties with high ornamental value and edible large fruit.

M. Robusta is a species of Malus native to northern Hebei, China, and is a typical apple rootstock and ornamental plant in northern China. Through long-term natural and artificial selection, M. Robusta has been selected for a spreading habit with strong vigor, bright white flowers, a large number of flowers, abundant pollen, dark green leaves, high seed germination rate, and especially large fruit relative to common crabapple cultivars; it also has eight marked longitudinal convex arrises on fruit surface, appearing the irregular octagon shape, hence its name as 'eight arrises crabapple' in the local area. Moreover, this plant has a wide range of environmental adaptability and high cold $\left(-37^{\circ} \mathrm{C}\right)$, drought, and disease resistance $(\mathrm{Li}, 2001)$. The fruit is also used in fresh and processed foods. As an ornamental plant, it is desirable for breeders to have red or pinkish-white flowers and fullred fruits, while maintaining high cold, drought, and disease resistance and adaptability (Zhang et al., 2020; Zhou et al., 2020).

We found a new cultivar with red flower petals at the balloon stage and pinkish-white petals at the beginning of flowering, followed by fruits with red skin. Malus Robusta 'Hongbaleng' was derived from a natural mutant of the M. Robusta offspring group, and its red-skin fruit and large fruit shape distinguish it from its maternal plant and other crabapple cultivars. These flower and fruit properties were found to be stable and consistent during a 3-year observation period, and they may be important traits for growers to enhance their economic income. Although there are many red-flowered and red-fruited ornamental crabapple varieties, the edible varieties with large full-red fruits are rare. In addition, 'Hongbaleng' is an ideal material for the study of fruit coloration and fruit development due to its full red pericarp ( $\mathrm{Lu}$ et al., 2017; Zhang et al., 2015). The results are expected to provide a technical reference to improve directional breeding of ornamental crabapples as well as red fruit coloration in other plants.

\section{Origins}

We collected more than 1200 seeds from a 20-year-old M. Robusta plant and sowed them on raised beds in the field in Mar. 2000 near Bangshuiyu village, Yanqing District of Beijing, China, then a full red-skin fruit mutation in M. Robusta was first observed in 2004. The field is located at $40.36^{\circ} \mathrm{N}$ and $115.97^{\circ} \mathrm{E}$, and the average annual temperature is $8{ }^{\circ} \mathrm{C}$, and the average altitude is more than $500 \mathrm{~m}$. After 4 years of observation, we selected the full red-skin fruit mutants and propagated them by bud grafting in Sept. 2004 and shoot grating in Apr. 2005 using M. Robusta and M. hupehensis as 3-year-old rootstocks, respectively. In 2006 and 2008, 206 grafted plants showed the same flowering and fruiting characteristics as their maternal plant and were then named M. 'Hongbaleng'. From 2009 to 2013, we also conducted regional experiments in Changping, Shunyi, and Yanqing districts. 'Hongbaleng' displayed the same tree habit and flowering and fruiting characteristics in these places. On 5 Dec. 2014, the new cultivar was designated $M$. 'Hongbaleng' by the Beijing Forest Variety Examination and Approval Committee (accession no. S-SV-MP-029-2014).

\section{Description}

Morphological traits of $M$. 'Hongbaleng' were evaluated according to the guidelines for the conduct of tests for Distinctness, Uniformity and Stability (DUS) (Liu, 2018; UPOV, 2003) and compared with those of the mother parent M. Robusta as the control (CK). 0 These plants were 10 years old and grown on the popular M. Robusta and $M$. hupehensis rootstock in The Ornamental Crabapple Germplasm Resource Garden of Beijing University of Agriculture, Yanqing 
$\mathbf{A}$
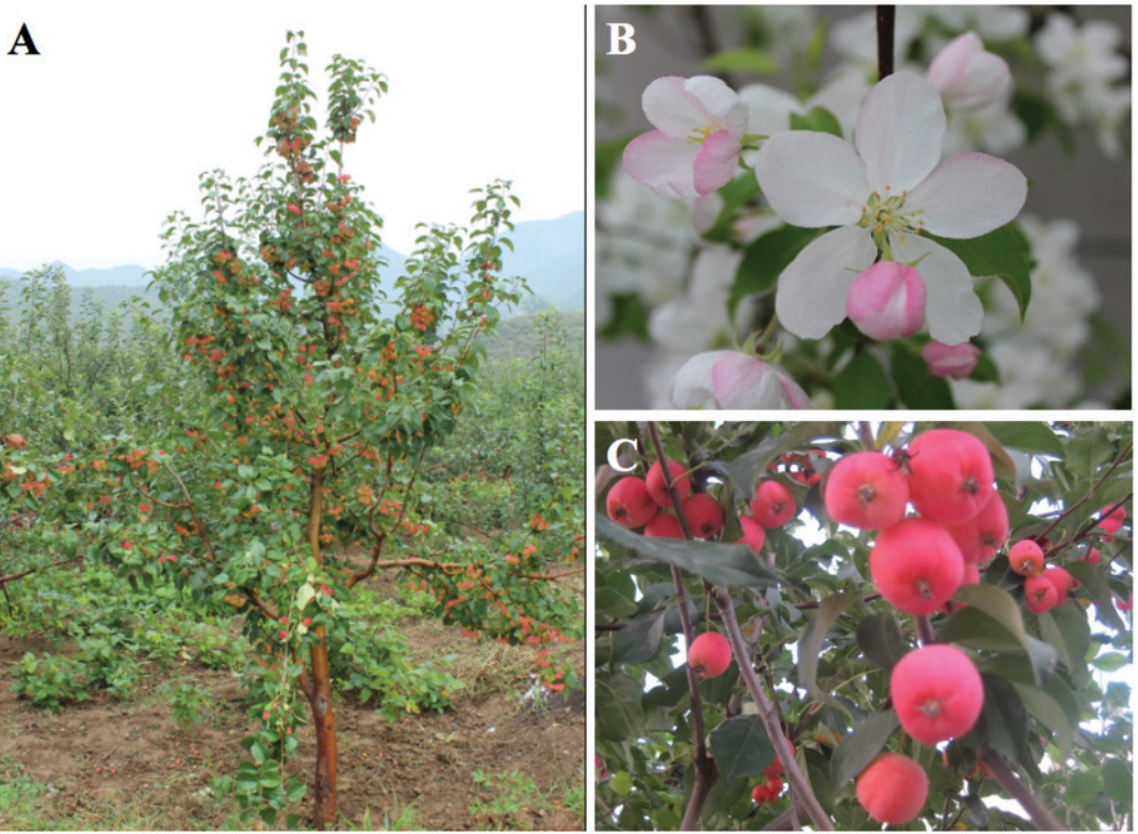

Fig. 1. Phenotypic characteristics of $M$. 'Hongbaleng' crabapple cultivar. (A) Upright growth habit at fruit-ripening stage (5 Sept. 2014). (B) Balloons and opened flowers (21 Apr. 2014). (C) Full red fruits (9 Sept. 2014). The photographs were taken in Bangshuiyu, Yanqing District, Beijing, China.

District of Beijing. For the five plants tested, 30 branches $\approx 20 \mathrm{~cm}$ long from the south side of the upper-middle and lower canopy per plant were selected and investigated for plant morphological traits. The sampling times were arranged as follows: young leaves in early May, mature leaves in mid-June, leaf blades (main color just before leaf fall) in late October, flowers in mid-April, and fruits in late September. We used a group data comparison analysis to compare the different traits of the new cultivar and CK plant via a $t$ test in which the length, diameter, and transverse and longitudinal diameters of the trunks, branches, leaves, flowers, and fruits were all measured with a ruler or Vernier caliper. All references to color were based on the Royal Horticultural Society (2007; RHS, fifth ed., UK) color chart. The fruit soluble solids, titratable acid, and vitamin $\mathrm{C}(\mathrm{Vc})$ content, and fruit hardness across 10 samples $(n=10)$ were determined by refractometry, titration, spectrophotometry, and durometry, respectively (Shen et al., 2011).

Tree. M. 'Hongbaleng' exhibits an upright habit and vertical ramose crown with strong vigor (Fig. 1A). Annual shoots are grayorange (RHS 165A) with a moderate number of lenticels and more pubescents, relative to CK plants. Perennial branches are graybrown (RHS N199C) with strong branching. The shoot length and the internode length are shorter in $M$. 'Hongbaleng' plants than in CK plants, suggesting that $M$. 'Hongbaleng' has a compact and dwarfed phenotype relative to its maternal parent, which is beneficial to easier management (Table 2).

Flower. M. 'Hongbaleng' has umbellate inflorescences with five to six single flowers (Fig. 2A). The flower petals base partly overlapping at an average of five petals per flower, which exhibit a broad elliptical shape (Table 1). The flower balloon is a red-purple (RHS 68A) (Fig. 2A), and petals that are not fully unfolded at the first bloom are pinkishwhite (RHS N57D) (Fig. 2B and C). When flowers are in full bloom, both the inner side and outer sides of the petals are white (RHS 155D) (Fig. 2D), with an average length of $1.91 \mathrm{~cm}$ and width of $1.52 \mathrm{~cm}$, but a few petals have a basal zone on the inner side of $M$. 'Hongbaleng' that are white (RHS 155D); additionally the color of the marginal zone of the inner side of the petal is pinkish-red (RHS N57D), and that of the outer side is purple-red (RHS-N57A). These values are somewhat different between the marginal and middle zones of the inner side and outer side of the petals (Fig. 1B). The diameter of the blooming flowers exhibits a shallow cup-like shape and is $4.28 \mathrm{~cm}$ on average, with a nonprominent petal vein. The length of the pedicel is on average $4.67 \mathrm{~cm}$, and the diameter of the pedicel is $\approx 0.7 \mathrm{~mm}$ (Table 1). All of these investigations were conducted at the full blooming stage, except for flower balloon coloration. These observations suggest that the petal coloration of $M$. 'Hongbaleng' in the initial opening and blooming stage differs significantly from that of its parent CK plant. Meanwhile, the filaments are more numerous, and the stamens length and the bud size are both larger, but the petal length is shorter in $M$. 'Hongbaleng' plants than in its CK plants (Table 1). These results indicate that the initial floral morphology, such as large buds, red petals, and abundant filaments, is a significant variation characteristic that distinguishes $M$. Hongbaleng from its parent, which not only strengthens the ornamental value of this new cultivar but also provides selected material for further floral color breeding.

Foliage. The upper side of the young leaves appear light green (RHS 143C) in the early spring, and the mature leaves are deep green (RHS 137B). The main color before leaf fall is yellow green (RHS 145A). The mature leaf shape is elliptical with an average length of $5.53 \mathrm{~cm}$ and width of $3.47 \mathrm{~cm}$; the length-towidth ratio is equal to 1.59 on average, and the single leaf area is $13.43 \pm 0.98 \mathrm{~cm}^{2}$ on average. The glossiness on the upper side of the leaf blade is strong, and the leaf tip is acuminate, smooth, and curved with obtuse serrate margins of the leaf edge. The petiole length and diameter are 2.05 and $0.15 \mathrm{~cm}$ on average, respectively. The stipule is always present, with an average length of $1.27 \mathrm{~cm}$ and width of $\approx 0.12 \mathrm{~cm}$. The length and width of the leaves and stipules, single leaf area, and leaf length/ width ratio are smaller in the new cultivar plants than those in the CK plants (Table 2).

Fruit. The fruit is globose and conical with an average longitudinal scale of $2.24 \mathrm{~cm}$ and a transverse scale of $2.68 \mathrm{~cm}$, and the average weight of a single fruit is $9.07 \mathrm{~g}$ (Table 1). The peel of mature fruits is yellow (RHS $8 \mathrm{C}$ ) and $100 \%$ red (RHS 33C) on the fruit surface (Fig. 1C). The large fruit peel color of $M$. 'Hongbaleng' changed during the fruit development period. The baby fruit peel
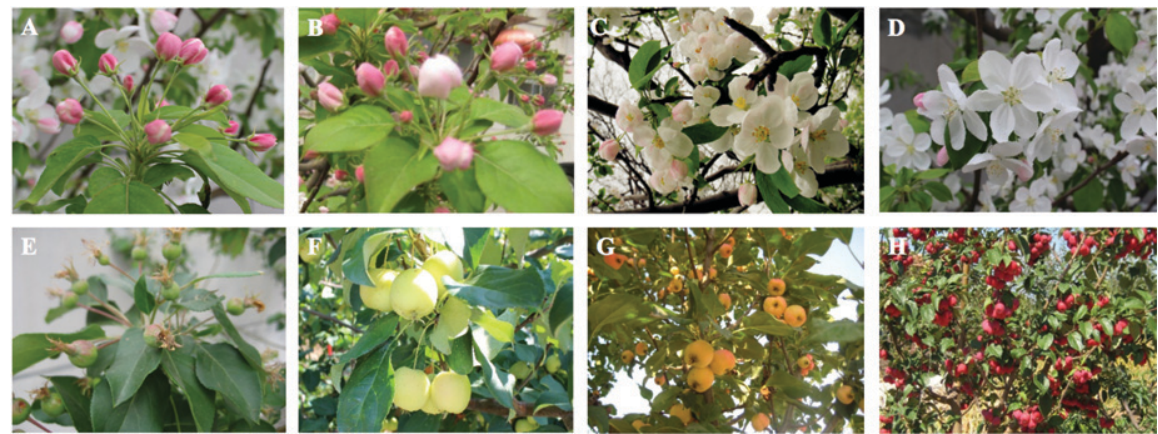

Fig. 2. Characteristics of flower and fruit of $M$. 'Hongbaleng'. (A) Small balloon stage (15 Apr.). (B) Big balloon stage (18 Apr. 2014). (C) Beginning of flowering stage (20 Apr. 2014). (D) Full-bloom stage (23 Apr. 2014). (E) Young fruit stage (5 May 2014). (F) Color-turning stage I (green to white transition period) (15 July 2014). (G) Color-turning stage II (yellow to red transition period) (25 Aug. 2014). (H) Ripening and full red fruits (25 Sept. 2014). The photographs were all taken in Bangshuiyu, Yanqing District, Beijing, China. 
Table 1. Comparisons of the flower and fruit traits between parent M. Robusta and offspring $M$. 'Hongbaleng'.

\begin{tabular}{|c|c|c|}
\hline Comparison items & M. Robusta & M. 'Hongbaleng' \\
\hline \multicolumn{3}{|l|}{ Flower } \\
\hline Petal shape & Broad elliptic & Broad elliptic \\
\hline Flower type & Single & Single \\
\hline Flower diameter $(\mathrm{cm})$ & $4.15 \pm 0.24$ & $4.28 \pm 0.12 \mathrm{~ns}$ \\
\hline Flower shape & Shallow cup & Shallow cup \\
\hline Petal position & Free & Overlapping \\
\hline Petal veins & Not prominent & Not prominent \\
\hline Unopened flower color & Red purple (RHS 63B) & Red purple (RHS N57D) \\
\hline Petal color & White (RHS 155D) & White (RHS 155D) \\
\hline Petal length $(\mathrm{cm})$ & $2.28 \pm 0.12$ & $1.91 \pm 0.11 \mathrm{~s}$ \\
\hline Petal width $(\mathrm{cm})$ & $1.32 \pm 0.15$ & $1.52 \pm 0.14 \mathrm{NS}$ \\
\hline Large buds/umbel & $4.1 \pm 0.12$ & $6.0 \pm 0.33 \mathrm{~s}$ \\
\hline No. of petals/flower & $5 \pm 0.00$ & $5.0 \pm 0.00 \mathrm{NS}$ \\
\hline Pedicel length $(\mathrm{cm})$ & $4.43 \pm 0.56$ & $4.67 \pm 0.45 \mathrm{NS}$ \\
\hline Pedicel diameter $(\mathrm{cm})$ & $0.068 \pm 0.04$ & $0.07 \pm 0.05 \mathrm{NS}$ \\
\hline Filament length $(\mathrm{cm})$ & $0.92 \pm 0.06$ & $0.73 \pm 0.04 \mathrm{NS}$ \\
\hline No. of filaments & $19.40 \pm 2.88$ & $21.00 \pm 2.12 \mathrm{~s}$ \\
\hline Stamen length $(\mathrm{cm})$ & $0.88 \pm 0.03$ & $1.07 \pm 0.07 \mathrm{~s}$ \\
\hline \multicolumn{3}{|l|}{ Fruit } \\
\hline Fruit size & Large & Large \\
\hline Fruit hardness $\left(\mathrm{kg} / \mathrm{cm}^{2}\right)$ & $10.24 \pm 1.08$ & $11.62 \pm 0.09 \mathrm{~s}$ \\
\hline Fruit shape & Ellipsoid conical & Globose conical \\
\hline Fruit calyx & Always present & Always present \\
\hline Pedicel cavity & Narrow & Wide \\
\hline Calyx cavity & Shallow & Shallow \\
\hline Soluble solids (\%) & $15.2 \pm 1.09$ & $18.3 \pm 1.12 \mathrm{HS}$ \\
\hline Fruit setting rate/umbel & $5.5 \pm 0.23$ & $6.3 \pm 0.17 \mathrm{~s}$ \\
\hline $\begin{array}{l}\text { Titratable acid }(\mathrm{mmol} / 100 \mathrm{~g}) \\
\quad(\mathrm{mg} / 100 \mathrm{~g})\end{array}$ & $2.8 \pm 0.21$ & $2.1 \pm 0.14 \mathrm{~s}$ \\
\hline $\mathrm{Vc}(\mathrm{mg} / 100 \mathrm{~g})$ & $4.0 \pm 0.91$ & $4.4 \pm 0.88 \mathrm{NS}$ \\
\hline Baby fruit color & Yellow green (RHS 144D) & Yellow green (RHS 144D) \\
\hline Peel color & Yellow (RHS 11C) & Yellow (RHS 8C) \\
\hline Cover color & Orange red (RHS N34C) & Red (RHS 33C) \\
\hline Fruit longitudinal scale $(\mathrm{cm})$ & $1.42 \pm 0.21$ & $2.24 \pm 0.18 \mathrm{~s}$ \\
\hline Fruit transverse scale $(\mathrm{cm})$ & $1.39 \pm 0.09$ & $2.68 \pm 0.12 \mathrm{~s}$ \\
\hline Fruit shape index & $1.02 \pm 0.08$ & $0.835 \pm 0.02 \mathrm{NS}$ \\
\hline Single fruit weight $(\mathrm{g})$ & $6.64 \pm 1.02$ & $9.07 \pm 0.08 \mathrm{HS}$ \\
\hline Length of stalk $(\mathrm{cm})$ & $4.23 \pm 0.23$ & $3.64 \pm 0.18 \mathrm{~s}$ \\
\hline Diameter of stalk $(\mathrm{cm})$ & $0.085 \pm 0.05$ & $0.106 \pm 0.05 \mathrm{~s}$ \\
\hline Pulp texture & Crisp & Crisp \\
\hline
\end{tabular}

Data are shown as means \pm SE. The significant differences between $M$. Robusta and $M$. 'Hongbaleng' were determined by Student's $t$ test $(P<0.05, P<0.01)$. HS = highly significant; $\mathrm{s}=$ significant; $\mathrm{NS}=$ not significant. Qualitative descriptions were conducted according to the International Union for the Protection of New Varieties of Plants (UPOV, 2003).

is green (RHS 145A) (Fig. 2E), and the fruit peel turns whitish green (RHS 144D) in midJuly (Fig. 2F) then yellow (RHS 11A) in early August. The covering color on the fruit peel surface is red (RHS 33C) on the sunny side in late August (Fig. 2G), and then exhibits full red coverage in early September, with maturity in late September (Fig. 2H). The length and diameter of the fruit stalk are 3.64 $\mathrm{cm}$ and $0.106 \mathrm{~cm}$ on average, respectively. On the surface of the fruit peel, there is strongly expressed glossiness, covering slightly waxy powder covering, obvious ridges, and light fragrance. The flesh is yellowish-white and crispy. The calyx is always present with a shallow concave calyx cavity (Table 1). The pedicel cavity is shallow and the width is concave. The fruit-setting rate is high and fruit persistence is long until flowers bloom the following year. The fruit hardness is $\approx 11.62 \mathrm{~kg} / \mathrm{cm}^{2}$, and the soluble solid content, titratable acid content and $\mathrm{Vc}$ content in the flesh at the equator of the fruit are 18.3 $\mathrm{mg} / 100 \mathrm{~g}, 2.1 \mathrm{mmol} / 100 \mathrm{~g}$, and $4.4 \mathrm{mg} / 100$ $\mathrm{g}$, on average, respectively (Table 1 ). All of the preceding investigations were conducted flowering in Beijing and northern Hebei Province. Thereafter, the leaves spread, and the shoots grow in mid-May. Young fruits grow in early May, and the fruit surface is initially yellow-green, turns red on the sunny side in mid-August, and exhibits full-red coverage fruits in the early September, with maturity in late September. Then, the pale green or slightly yellow-green leaves fall in the middle of November. Compared with its parent, $M$. 'Hongbaleng' has a long, big, red floral balloon stage, an early fruit color-turning stage, and a long fruit coloring stage, as well as a long autumn-colored leaf stage, extending the ornamental time and enhancing the ornamental quality.

\section{Cultivation and Pest Management}

M. 'Hongbaleng' has strong adaptability and is suitable for cultivation in Beijing and northern China, as well as in regions with similar climatic conditions. It is propagated using grafting onto rootstocks such as $M$. Robusta and $M$. hupehensis by selecting healthy, full buds and branches from the cultivar as the scion. This process also facilitates early shaping through the grafting of the scion onto the perennial rootstocks. The grafted plants $>3$ years old with a diameter $>2.5 \mathrm{~cm}$ were transplanted at 800-1000/ $666.67 \mathrm{~m}^{2}$ in the nursery. We recommend that $M$. 'Hongbaleng' trees be transplanted according to the American Standard for Nursery Stock (American Horticulture Industry Association, 2014). All trees should be healthy with tops of good quality (i.e., leaf color appropriate for cultivar and no apparent injury), vigorous, and well-rooted with growth normal for the species or variety (e.g., reasonably straight trunks according to the habit of growth). We also recommend that $M$. 'Hongbaleng' trees used for landscape construction be transplanted with $1 \mathrm{~m} \times 2 \mathrm{~m}$ plant spacing (both plant and row) and shaped and trained early in the nursery for future ornamental applications. The trees used for fruits shall be transplanted at $1 \mathrm{~m}$ $\times 3 \mathrm{~m}$ or $1 \mathrm{~m} \times 4 \mathrm{~m}$ plant spacing and row spacing, respectively.

For the $M$. 'Hongbaleng' root and the root ball, not less than three root branches should be present within $12.5 \mathrm{~cm}$ from the collar, and the root shall carry the minimum caliper of the grade for not less than $15 \mathrm{~cm}$ below the collar. When digging up the tree, if the trunk diameter at $15 \mathrm{~cm}$ interval above the ground level is 2.5 to $5 \mathrm{~cm}$, a root ball of 45 to $60 \mathrm{~cm}$ diameter and 30 to $40 \mathrm{~cm}$ depth is dug out and then wrapping in a 30 to $40 \mathrm{~cm}$ ground fabric bag; if the tree trunk diameter is 5 to $10 \mathrm{~cm}$, a root ball of 60 to $100 \mathrm{~cm}$ diameter and 40 to $65 \mathrm{~cm}$ depth is dug out, and then wrapping in a 40 to $65 \mathrm{~cm}$ ground fabric bag. When the tree trunk diameter is more than $10 \mathrm{~cm}$, we dig out the root ball at more than $100 \mathrm{~cm}$ diameter and $80 \mathrm{~cm}$ depth, with $100 \mathrm{~cm}$ ground fabric bag. The center of the plant trunk should be in the center of the root ball. When these large trees are transplanted, a suitable planting hole or ditch should be prepared according to the size of the transplanted root ball above (American Horticulture 
Table 2. Comparisons of plant habit and leaf traits between parent M. Robusta and offspring $M$. 'Hongbaleng'.

\begin{tabular}{|c|c|c|}
\hline Comparison items & M. Robusta & M. 'Hongbaleng' \\
\hline \multicolumn{3}{|l|}{ Tree } \\
\hline Tree habit & Upright & Upright \\
\hline Tree vigor & Strong & Strong \\
\hline Tree crown shape & Ramose crown & Ramose crown \\
\hline \multicolumn{3}{|l|}{ Shoots } \\
\hline Shoot length $(\mathrm{cm})$ & $59.05 \pm 1.03$ & $48.30 \pm 0.91 \mathrm{HS}$ \\
\hline Shoot diameter $(\mathrm{cm})$ & $1.47 \pm 0.06$ & $1.63 \pm 0.07 \mathrm{NS}$ \\
\hline Internode length $(\mathrm{cm})$ & $3.16 \pm 0.12$ & $2.28 \pm 0.09 \mathrm{~s}$ \\
\hline Shoot color & Gray-orange (RHS 165A) & Gray orange (RHS 165A) \\
\hline Perennial branch color & Gray-orange (RHS 165A) & Gray brown (RHS N199C) \\
\hline Lenticels number & Many & Medium \\
\hline Pubescence & Few & Very many \\
\hline Branching & Strong & Strong \\
\hline \multicolumn{3}{|l|}{ Leaves } \\
\hline Color of leaf blade & Light green (RHS N144C) & Light green (RHS 143C) \\
\hline Leaf color just before leaf fall & Yellow green (RHS 146C) & Yellow green (RHS 145A) \\
\hline Color of leaf blade & Green (RHS 137B) & Green (RHS 137B) \\
\hline Leaf shape & Elliptic & Elliptic \\
\hline Petiole length $(\mathrm{cm})$ & $2.02 \pm 0.03$ & $2.14 \pm 0.02 \mathrm{NS}$ \\
\hline Petiole width $(\mathrm{cm})$ & $0.18 \pm 0.01$ & $0.15 \pm 0.01$ \\
\hline Leaf blade lobes & Present & Always present \\
\hline Leaf margin & Serrate & Obtuse serrate \\
\hline Leaf glossiness & Medium & Medium \\
\hline Leaf length $(\mathrm{cm})$ & $9.60 \pm 0.26$ & $5.53 \pm 0.24 \mathrm{HS}$ \\
\hline Leaf width $(\mathrm{cm})$ & $5.51 \pm 0.13$ & $3.47 \pm 0.18 \mathrm{HS}$ \\
\hline Leaf length/width ratio & $1.74 \pm 0.06$ & $1.59 \pm 0.12 \mathrm{NS}$ \\
\hline Stipule length (cm) & $1.76 \pm 0.05$ & $1.27 \pm 0.07 \mathrm{~s}$ \\
\hline Stipule width $(\mathrm{cm})$ & $0.48 \pm 0.02$ & $0.12 \pm 0.02 \mathrm{HS}$ \\
\hline GUSL & Medium & Strong \\
\hline Leaf area $\left(\mathrm{cm}^{2}\right)$ & $24.64 \pm 1.24$ & $13.43 \pm 0.98 \mathrm{HS}$ \\
\hline Leaf tip shape & Acuminate & Acuminate \\
\hline
\end{tabular}

Data are shown as means \pm SE. GUSL $=$ glossiness of upper side of the leaf blade. The significant differences between $M$. Robusta and $M$. 'Hongbaleng' were determined by Student $t$ tests $(P<0.05$, $P<0.01) . \mathrm{s}=$ significant; NS $=$ not significant. Qualitative descriptions were conducted according to the International Union for the Protection of New Varieties of Plants (UPOV, 2003).

Industry Association, 2014). The planting ditch or the planting hole shall be dug at least $1 \mathrm{~m}$ deep and $1.5 \mathrm{~m}$ wide for organic fertilizer and borrowed soil if there are poor conditions. The organic fertilizer should be evenly mixed with the topsoil dug out of the planting ditch, backfilled into the planting hole, and compacted to fill with sufficient water to improve the survival rate and shorten the slow seedling period (Beijing Gardening and Greening Bureau, 2008). The organic fertilizer is mixed as Farmyard manure $100 \mathrm{~kg}$, Bacillus subtilis $0.1 \mathrm{~kg}$, ABT rooting power $50 \mathrm{mg}$, urea $(\mathrm{N}) 300 \mathrm{~g}$, and $\mathrm{KH}_{2} \mathrm{PO}_{4} 100 \mathrm{~g}$ for transplant.

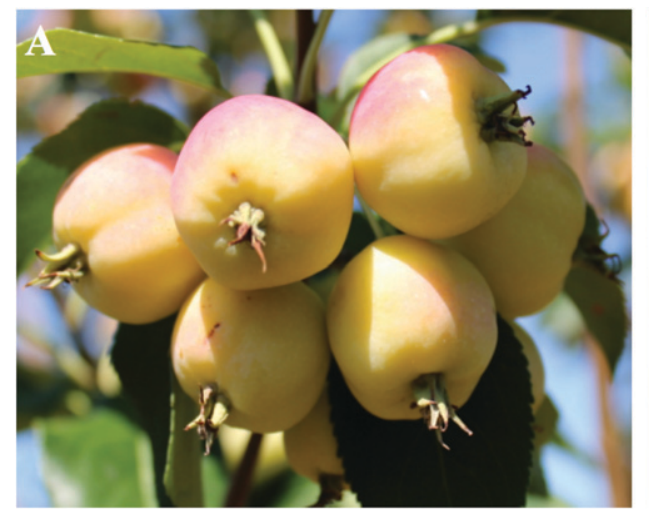

Fig. 3. Comparison of the fruit traits between parent M. Robusta (A) and M. 'Hongbaleng' (B). The photographs were taken in Bangshuiyu, Yanqing District, Beijing, China, on 25 Sept. 2014.

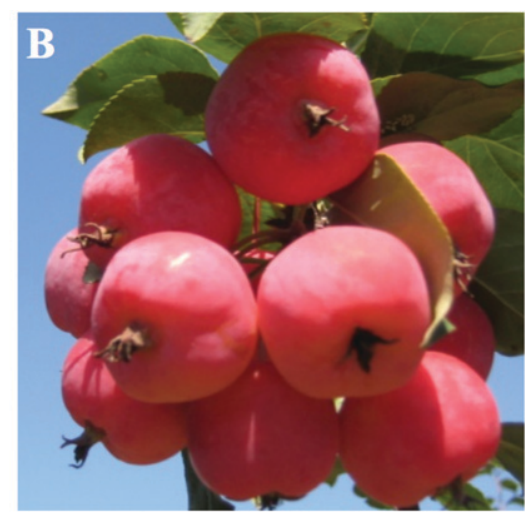

and remove overlapping branches and cross branches to improve light conditions and promote the early formation of tree crowns in the adult stage (Fumey et al., 2008).

No significant plant diseases or insect pests were observed in natural planting of this cultivar, but early attention should be given to apple canker, protecting the wound when pruning, applying white lime aqueous solution on the tree trunk in late autumn, and to spraying a $5^{\circ} \mathrm{BE}$ lime sulfur mixture before germination (Sikdar et al., 2018). Aphids and spider mites were occasionally found and controlled by spraying pesticide, and sporadic Carposina niponensis was controlled by applying a sex pheromone (Beijing Gardening and Greening Bureau, 2015). Juniperus sabina plants should be kept away from crabapple plants to avoid rust (Zhang et al., 2020). This variety is suitable for planting and use in garden landscapes and for afforestation, as well as apple rootstock and pollinizers for apple cultivation in northern China and for breeding the new varieties of ornamental crabapple.

\section{Availability}

M. 'Hongbaleng' is available from the Yanqing District Fruit Service Center and Beijing Hengcheng Tongtai Agricultural Science and Technology Co., Ltd., and Hebei Yutangyuan Ecological Agriculture Co., Ltd.

\section{Literature Cited}

American Horticulture Industry Association. 2014. American standard for nursery stock. ANSI Z60:1.

The desired diameter and height of $M$. 'Hongbaleng' trees at $1.0,1.5$, and $2.5 \mathrm{~cm}$ or greater trunk diameter corresponds to a 100 , 150 , and $180 \mathrm{~cm}$ tree height, respectively. The tree at $0.8 \mathrm{~cm}$ and larger single trunk diameter shall be branched and those at 1.5 $\mathrm{cm}, 2 \mathrm{~cm}$, and $2.5 \mathrm{~cm}$ single trunk diameter shall have three or more branches. We recommend that these trees shall be pruned back to multiply the branching structure and develop a more formal effect when the tree is young (American Horticulture Industry Association, 2014). It is advisable to cut lightly, retain more branches, open the branch angle,

Beijing Gardening and Greening Bureau. 2008. Seeding production of fruit trees (in Chinese). DB11/T560-2008.

Beijing Gardening and Greening Bureau. 2015. Technical specification for propagation and cultivation of ornamental crabapple (in Chinese). DB11/1243-2015.

Dudash, M.R., C. Hassler, P. Stevens, and C. Fenster. 2011. Experimental floral and inflorescence trait manipulations affect pollinator preference and function in a hummingbird-pollinated plant. Amer. J. Bot. 98:275-282, doi: 10.3732/ajb.1000350.

Fumey, D., P.E. Lauri, Y. Gueìdon, C. Godin, and E. Costes. 2008. Effects of pruning on the apple tree: From tree architecture to modeling. International Symposium on Integrating Canopy, Rootstock and Environmental Physiology in Orchard Systems. Geneva, New York, United States, doi: 10.13140/2.1.3831.9364.

Henry, D.G. 2007. Crabapple cultivars tested as street trees: third report. Arboric. Urban For. 33(3):176-181.

Li, Y. 2001. Research of germplasm resources of Malus Mill (in Chinese). China Agriculture Press, Beijing, China.

Liu, Y. 2018. Studies of Standard Description and Database Construction of Malus Cultivars (in Chinese). Chinese Academy of Forestry, Beijing, China.

Lu, Y., Y. Bu, S. Hao, Y. Wang, J. Zhang, J. Tian, and C. Yao. 2017. MYBs affect the variation in the ratio of anthocyanin and flavanol in fruit peel and flesh in response to shade. J. Photochem. Photobiol. B 168:40-49, doi: 10.1016/j. jphotobiol.2017.01.017. 
Qian, G. and G. Tang. 2005. A review on the plant taxonomic study on the genus Malus Miller (in Chinese). J. Nanjing Forestry Univ. (Natural Sciences Edition) (03):94-98.

Qian, G. 2005. Taxonomic study of Malus Mill (in Chinese). Nanjing Forestry University, Nanjing, China, doi: 10.7666/d.y749343.

Royal Horticultural Society. 2007. RHS color chart. 5th ed. Royal Horticultural Society, London, UK.

Shen, H., T. Zhao, T. Song, Y. Yao, and J. Gao. 2011. Genetic diversity analysis in natural hybrid progeny of ornamental crabapple, Malus 'Royalty'.
Acta Hort. Sinica 38(11):2157-2168, doi: 10.16420/j.issn.0513-353x.2011.11.017.

Sikdar, P., M. Willett, and M. Mazzola. 2018. Pruning of Manchurian crabapple for management of speck rot and Sphaeropsis rot in apple. HortScience 53(3):329-333, doi: 10.21273/ HORTSCI12672-17.

UPOV. 2003. Guidelines for the conduct of tests for distinctness, uniformity and stability [ornamental apple (Malus Mill.)]. TG/192/1.

Wang, Y. 1989. Cultivation techniques of $M$. Robusta (in Chinese). Hebei Fruits (02): 10-12.
Zhang, L., Y. Mao, Y. Wang, L. Yang, Y. Yin, X. Shen, C. Zhang, and D. Zhang. 2020. Malus spectabilis 'Duojiao': a new yellow-leaf cultivar. HortScience 55(7):1155-1158, doi: 10.21273/HORTSCI14865-20.

Zhang, M., H. Chen, H. Wang, A. Shi, P. Hong, and K. Liu. 2015. Status and prospect of plants' distant hybridization breeding (in Chinese). J. Anhui Agr. Sci. 43(1):11-17.

Zhou, T., H. Jiang, D. Zhang, J. Fan, L. Zhang, G. Wang, W. Zhang, and F. Cao. 2020. 'Zi Dieer' crabapple. HortScience 55:272-274, doi: 10.21273/HORTSCI14590-19. 